\title{
KATRIN, an experiment for determination of the $v$-mass: status and outlook
}

\author{
Michael Sturm* ${ }^{* \dagger}$ \\ Karlsruhe Institute for Technology \\ Postfach 3640, 76021 Karlsruhe, Germany \\ E-mail: michael.sturmekit.edu
}

\begin{abstract}
Recent results of the measurement of the cosmic microwave background fit remarkably well with the simplest cosmological $\Lambda \mathrm{CDM}$ model. Observations on small (galactic) scales can be explained by a warm dark matter model (WDM), while the current $\Lambda$ CDM model seems to fail. In this context $\mathrm{eV}$ or even $\mathrm{keV}$ sterile neutrinos are getting again in vogue. Therefore one of the most fundamental tasks over the next years will be the determination of the absolute mass scale of neutrinos. Model-independent results are only provided by kinematic $\beta$-decay experiments. The Karlsruhe Tritium Neutrino experiment KATRIN aims at improving the sensitivity in the neutrino mass measurement down to $200 \mathrm{meV} / \mathrm{c}^{2}$ (90\%C.L.) and so will help to clarify the role of neutrinos in the early universe. KATRIN investigates spectroscopically the electron spectrum from tritium $\beta$-decay close to the kinematic endpoint of $18.6 \mathrm{keV}$. For such a precise mass determination a key parameter is the stability of the source in terms of $\beta$-activity and isotopic purity. For that reason KATRIN uses a strong windowless gaseous tritium source of almost pure molecular tritium (95\%) stabilised on $0.1 \%$ level with a throughput of $40 \mathrm{~g}$ tritium per day. The decay electrons are guided adiabatically from the source to the spectrometer by means of superconducting magnets while at the same time the tritium flow rate to the spectrometers has to be reduced by a factor $>10^{14}$. A tandem spectrometer system with an energy resolution of $\Delta \mathrm{E}=$ $0.93 \mathrm{eV}$ at $18.6 \mathrm{keV}$ is used for energy analysis, followed by a detector-system for counting the transmitted $\beta$-decay electrons. Both spectrometers are of the MAC-E-Filter (Magnetic Adiabatic Collimation followed by Electrostatic Filter) type and therefore combine a high energy resolution with a large luminosity. KATRIN is currently under construction at the Karlsruhe Institute of Technology (KIT) Campus North; it will prospectively start data taking in 2015. This talk will give an introduction to direct $v$-mass determination from tritium $\beta$-decay and an overview of the status of the main components of the KATRIN experiment.
\end{abstract}

VIII International Workshop on the Dark Side of the Universe,

June 10-15, 2012

Rio de Janeiro, Brazil

\footnotetext{
*Speaker.

${ }^{\dagger}$ for the KATRIN collaboration
} 


\section{Introduction}

The properties of neutrinos and especially their rest mass play an important role at the intersections of cosmology, particle physics and astroparticle physics. In standard cosmological models, our universe is filled with primordial neutrinos arising from freeze-out in the early universe. These neutrinos are natural candidates for non-baryonic hot dark matter [1]. A model-independent approach to determine the neutrino mass is the kinematical analysis of electrons from tritium $\beta$ - decay ${ }^{3} \mathrm{H} \rightarrow{ }^{3} \mathrm{He}+\mathrm{e}^{-}+\bar{v}_{e}$ near the endpoint energy $\mathrm{E}_{0}$ [2] where a non-vanishing neutrino mass $\mathrm{m}_{v}$ causes a distortion of the $\beta$-spectrum

$$
\frac{d^{2} N}{d E d t} \propto p\left(E_{0}+m_{e} c^{2}\right) \cdot\left(E_{0}-E\right) \cdot \sqrt{\left(E_{0}-E\right)^{2}+m_{v}^{2} c^{4}} \quad \text { with } m_{v}^{2}=\sum_{i=1}^{3}\left|U_{e i}^{2}\right| m_{i}^{2}
$$

This measurement allows a direct and model-independent determination of $\mathrm{m}_{v}$. Based on the results of the Mainz [3] and Troitsk [4] experiments an upper limit of $2 \mathrm{eV} / \mathrm{c}^{2}$ (95\% C.L.) was deduced [5]. The aim of the KArlsruhe TRItium Neutrino (KATRIN) experiment is the measurement of $\mathrm{m}_{v}$ with $200 \mathrm{meV} / \mathrm{c}^{2}$ design sensitivity (90\% C.L.) [6]. By improving the sensitivity by one order of magnitude in comparison to previous experiments, KATRIN will probe a cosmologyrelevant part of the neutrino mass parameter space.

\section{The KATRIN Experiment}

The effect of the neutrino mass is most pronounced in the endpoint region of the observed spectra as shown in figure 1. This also highlights a main challenge of the measurements, as in the case of tritium only $2 \cdot 10^{-13}$ of all decay electrons fall into the last $1 \mathrm{eV}$ of the distribution. The main requirements to set up a sensitive $\beta$-decay experiment are therefore a high overall source luminosity, high energy resolution and a very low background in order to gain sufficient statistics in the endpoint region. Besides the statistics of the measurement, challenges arise in the precise treatment of systematic effects like e.g. the inelastic scattering of electrons in the source volume.

The $70 \mathrm{~m}$ long KATRIN setup is shown in figure 2. KATRIN uses a strong windowless gaseous tritium source of almost pure molecular tritium (95\%). The decay electrons are guided adiabatically from the source through a transport section to the spectrometer system by means of superconducting magnets while at the same time the tritium flow rate to the spectrometers has to be reduced by

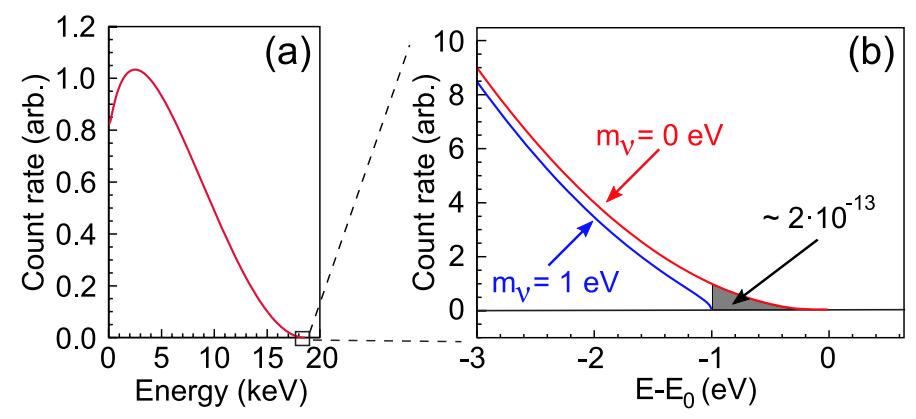

Figure 1: Energy distribution of electrons emitted in tritium $\beta$-decay 


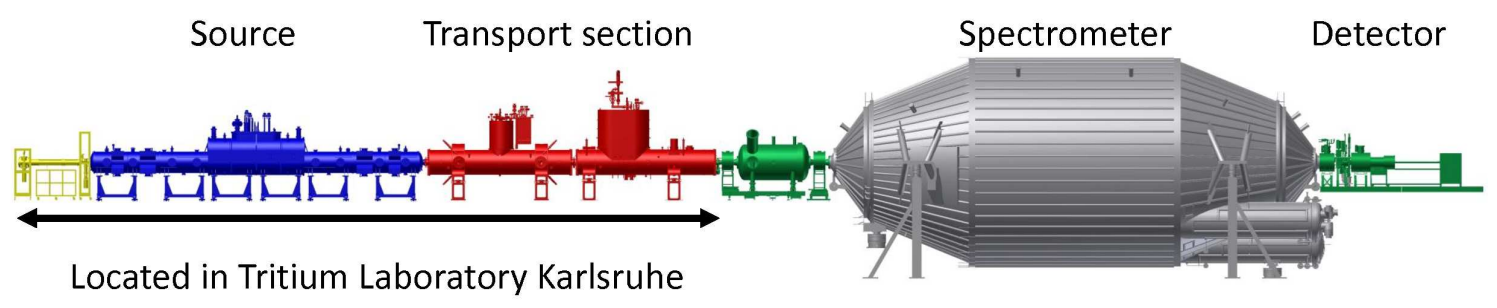

Figure 2: Overview of the KATRIN main beam-line. High purity $\mathrm{T}_{2}$ gas is being injected in the middle of the source tube with a flow rate of $1.8 \mathrm{mbar} 1 / \mathrm{s}\left(40 \mathrm{~g}\left(\mathrm{~T}_{2}\right) /\right.$ day $)$. Electrons from $\beta$-decay leave the source and are guided by magnetic fields through the transport section, while the remaining gas is being removed by active and cryogenic pumping. The pre-spectrometer filters out the low-energy part of the spectrum, thus only electrons close to the endpoint region can enter the main spectrometer for the precise energy analysis. Transmitted electrons are then detected by a low background Si-PIN detector system.

a factor $>10^{14}$, since the background rate generated by tritium decay within the spectrometers has to be less than $10^{-3}$ counts/s in order to reach the sensitivity KATRIN is aiming for. The transport section consists of a differential pumping section (DPS) and a cryogenic pumping section (CPS). In the DPS the tritium flow will be reduced by differential pumping while in the CPS tritium will be adsorbed on a pre-condensed argon layer prepared inside KATRIN's beam tube. In addition to the above mentioned low background an high energy resolution of the spectrometer is needed. As well as high statistics are a necessity. A tandem spectrometer system is used for energy analysis, followed by a detector-system for counting the transmitted $\beta$-decay electrons. Both spectrometers are of the MAC-E-Filter (Magnetic Adiabatic Collimation followed by Electrostatic Filter) [7], [8] type. In the spectrometer region, carefully designed electromagnetic field conditions have to be implemented. In particular, electromagnetic conditions leading to Penning-like trapping conditions have to be avoided in order to reach low background rates.

The KATRIN experiment is currently being built up at the site of the Tritium laboratory Karlsruhe. The following subsections address the details of some key components and their present status.

\subsection{Windowless Gaseous Tritium Source}

The KATRIN WGTS will basically consist of a tube of $10 \mathrm{~m}$ length and $90 \mathrm{~mm}$ diameter, kept at a cryogenic temperature of $30 \mathrm{~K}$, which is filled with molecular tritium gas of high isotopic purity $>95 \%$. One of the key parameters of KATRIN is the stability of the source on $0.1 \%$ level. Figure 3 shows the principle of KATRIN's Windowless Gaseous Tritium Source (WGTS). At the center of the beam tube molecular tritium gas will be injected continuously. After injection the $T_{2}$ molecules will diffuse to both ends of the WGTS beam-tube, where most of the tritium will be pumped out continuously by turbomolecular pumps (TMP), processed and reinjected. The density profile of the gas inside the $10 \mathrm{~m}$ long beam-tube has to be kept stable on the $0.1 \%$ level. Maintaining the required stable injection rate is provided by the Inner Loop System. In addition the temperature of the source beam tube has to be stabilised as well. Maintaining these conditions is a very challenging task. 


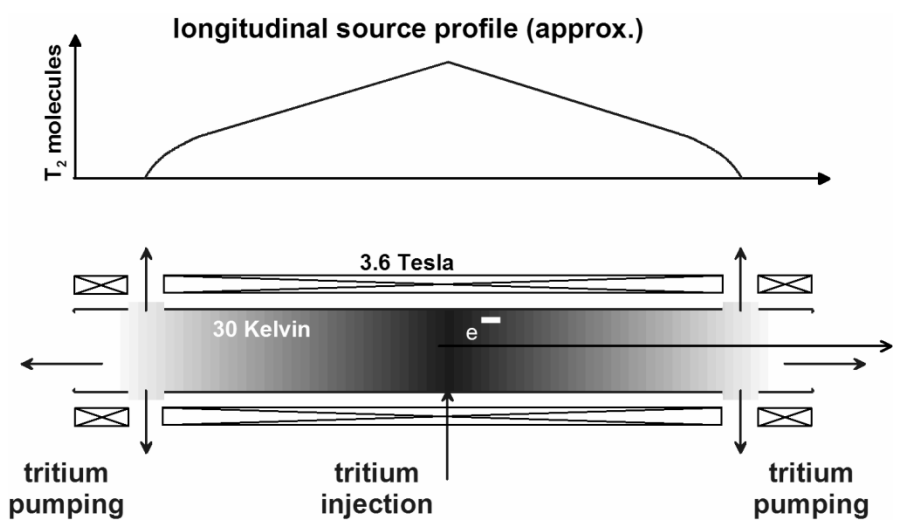

Figure 3: Principle of KATRIN's Windowless Gaseous Tritium Source.

\subsection{Temperature stabilisation}

To stabilise the beam tube temperature with a stability of $0.1 \%$ at a setpoint of about $30 \mathrm{~K}$, two copper tubes are braced on the source beam tube which directly couple the beam tube to a two phase Neon thermosiphon [9]. This cooling concept was succesfully tested at the WGTS demonstrator, a partly assembled version of the later WGTS cryostat which houses the later beam-tube and cryogenics but not the superconducting magnets. With the first measurements a temperature stabilisation in the milli-Kelvin range has been achieved. The temperature stability was found a factor 20 better than specified [10].

\subsection{Inner Loop System}

In order to keep the pressure profile stable it is necessary to inject the tritium gas with a stability of $0.1 \%$, concerning flow-rate and composition. This challenging task is performed by the Inner Loop System, illustrated in a simplified flow diagram in figure 4. Tritium is being injected from a pressure controlled buffer vessel over a capillary with constant conductivity in the middle of the source beam tube. The gas pumped out by the TMPs gets pumped through a palladium membrane filter (permeator) in a buffer vessel. From there the gas is led over a Laser Raman sampling cell and a regulating valve back into the pressure controlled buffer vessel. At the filter impurities like ${ }^{3} \mathrm{He}$ from tritium decay and tritiated methanes, being generated due to interactions of tritium with the carbon inside the stainless steel walls of the system, are being detached from the gas stream. The amount of gas not recirculated is replaced by tritium from TLK's Isotope Separation System.

The Inner Loop System has been set up and commissioned successfully. The first test-runs have been performed with a capillary of similar conductance as KATRIN's injection capillary and source tube [11]. Being designed for a stability of $10^{-3}$, these test runs showed that the loop system reaches a $2 \cdot 10^{-4}$ stability level during 4 week of continuous gas circulation.

\subsection{Laser Raman spectroscopy}

The precise knowledge of the composition of the tritium inlet gas of the WGTS is necessary to account for systematic effects in the WGTS, e.g. Doppler broadening, elastic scattering, nuclear recoil and the final state distribution of the $\left({ }^{3} \mathrm{HeT}\right)^{+}$daughter molecules. Laser Raman spectroscopy 


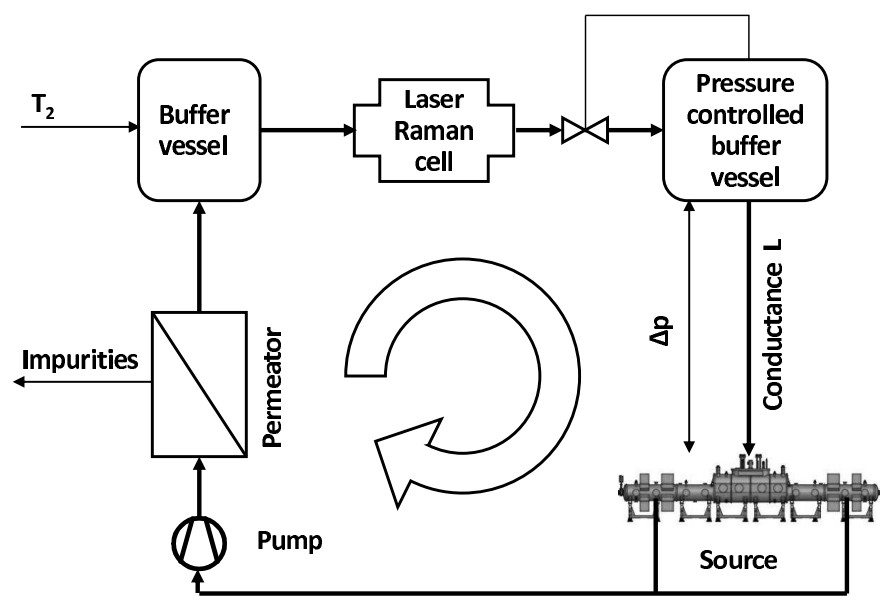

Figure 4: Simplified flow diagram of the Inner Loop System.

(LARA) is the method of choice for the monitoring of the gas composition in KATRIN since it allows the simultaneous monitoring of all hydrogen isotopologues $\left(\mathrm{T}_{2}\right.$, DT, HT, $\left.\mathrm{D}_{2}, \mathrm{HD}, \mathrm{H}_{2}\right)$ [12]. The test of the LARA system in the closed tritium loop LOOPINO during 3 weeks of non-stop operation showed that the required $0.1 \%$ precision is achieved under KATRIN-like conditions [13]. Further improvement of precision due to an optimisation of the laser beam path and the read-out mechanism of the optical detector are expected. Experimental tests of calculated Raman scattering cross-sections of all hydrogen isotopologues were made to improve the accuracy of the LARA measurements [14].

\subsection{Transport Section}

Adjacent to the WGTS the transport section guides the decay electrons to the spectrometer section by strong magnetic fields of $B=5.6 \mathrm{~T}$. Tritium flow reduction by the factor $10^{14}$ is achieved in two steps. First the differential pumping section DPS uses active pumping to provide tritium tritium flow reduction. The following cryogenic pumping section CPS uses cryo-sorption to achieve a further tritium flow reduction of $>10^{7}$. Here, the liquid helium cold inner beam-tube is covered with Argon frost, providing a large surface to cryo-sorp the tritium molecules. The CPS concept has been successfully tested in [16], [17]. The manufacturing of the CPS is ongoing and the delivery to KIT is expected by 2014 .

\subsection{Spectrometer and detector system}

Two electrostatic filters, based on the MAC-E principle, are used for energy analysis: The prespectrometer will reject most of the electrons which have energies less than about $300 \mathrm{eV}$ below the endpoint, which do not contain information on the anti-neutrino mass. The retarding potential of the main spectrometer will be varied to measure the spectrum in the last $30 \mathrm{eV}$ below the endpoint. The pre-spectrometer has been operated as a prototype for systematic investigations and hardware developments which are also relevant for the main spectrometer. A radon induced background signal, emerging from the material of vacuum getter strips, has been identified and suitable 
experimental measures for suppression were found [18]. The test operation of the prespectrometer is finished and it is ready for its final integration into the KATRIN setup.

The main spectrometer as MAC-E filter provides a resolution of $0.93 \mathrm{eV}$ for $18.6 \mathrm{keV}$ electrons. Since the main spectrometer has to operate at a background level of $10^{-3} \mathrm{cps}$, low-energy knock-on electrons created by cosmic muons in the vessel wall have to be rejected. For this not only a magnetic shielding is being relied upon, but also a UHV compatible wire electrode system [19] has been installed. At the moment preparations for baking out the main spectrometer vessel are ongoing.

The transmitted tritium decay electrons are counted by a detector based on a monolithic 148 pixel Si-PIN diode with an energy resolution of about $1 \mathrm{keV}$, and the ability to detect rates from $10^{-3} \mathrm{cps}$ in the end-point region and up to $10^{3} \mathrm{cps}$ during calibration runs, while keeping the background low. The focal plane detection system, developed by collaborating groups from the US, has been set up and is currently being tested at KIT. The commissioning and first measurements of the main spectrometer are scheduled for end of 2012 and beginning of 2013.

\section{Conclusion}

KATRIN has ambitious goals, both in particle physics and in the technical realization of the experimental set-up. The first measurements for the temperature stabilisation of the source beam tube with the demonstrator, as well as the first gas-flow reduction factor measurements with the DPS2-F have been successful. The Inner Loop system has been successfully commissioned and Laser Raman Spectroscopy for monitoring the gas composition has been successfully implemented. The next major steps to finalize the experiment setup will be finishing the manufacturing of the WGTS cryostat (expected for early 2015) and the delivery of the CPS (expected for early 2014). With the upcoming measurements at the main spectrometer in 2013 a main component of the experiment will be commissioned. With an estimated statistical uncertainty of $\Delta \mathrm{m}_{\text {stat }}^{2}=0.018$ $\mathrm{eV}^{2} / \mathrm{c}^{4}$ and a systematic uncertainty restricted to $\Delta \mathrm{m}_{\text {sys }}^{2}=0.017 \mathrm{eV}^{2} / \mathrm{c}^{4}$ this leads to the KATRIN design sensitivity of $\mathrm{m}_{v}<0.2 \mathrm{eV} / \mathrm{c}^{2}(90 \% \mathrm{CL})$ and the discovery potential of $\mathrm{m}_{v}=0.35 \mathrm{eV} / \mathrm{c}^{2}$ with $5 \sigma$ significance after 3 years of data taking ( 5 years real-time).

\section{References}

[1] S. Hannestad, Neutrinos in cosmology, New Journal of Physics, Vol 6, No. 108, 2004 arXiv:hep-ph/0404239v1

[2] E.W. Otten et al., Neutrino mass limit from tritium $\beta$-decay, Rep. Prog. Phys 71 (2008) 086201, arXiv:0909.2104

[3] Ch. Kraus et al., Final Results from phase II of the Mainz Neutrino Mass Search in Tritium $\beta$-Decay, Eur. Phys. J. C 40 (2005) 447-468.

[4] V.M. Lobashev et al., Direct search for mass of neutrino and anomaly in the tritium beta-spectrum, Phys. Lett. B 460 (1999) 227-235.

[5] J. Beringer et al. (Particle Data Group), Review of Particle Physics, Phys. Rev. D86, 010001 (2012)

[6] KATRIN Collaboration, KATRIN Design Report 2004, FZKA report 7090 
[7] G. Beamson et al., The collimating and magnifying properties of a superconducting field photoelectron spectrometer, Journal of Physics E: Scientific Instruments 13 (64-66) 1980

[8] A. Picard et al., A solenoid retarding spectrometer with high resolution and transmissin for keV electrons, Nuclear Instruments and Methods in Physics research Section B Volume 63, Issue 3, 1992, pages 345-358

[9] S. Grohmann et al., Stability analyses of the beam tube cooling in the KATRIN source cryostat, Cryogenics, Volume 49, Issue 8 (2009) 413-420

[10] S. Grohmann et. al., The thermal behaviour of the tritium source in KATRIN, submitted to Cryogenics

[11] M. Sturm, Dissertation, 2010, http://digbib. ubka.uni-karlsruhe.de/volltexte/1000019355

[12] M. Sturm et al., Monitoring of All Hydrogen Isotopologues at Tritium Laboratory Karlsruhe Using Raman Spectroscopy, Laser Phys. 202 (2010).

[13] S. Fischer et al., Monitoring of tritium purity during long-term circulation in the KATRIN test experiment LOOPINO using laser Raman spectroscopy, Fusion Sci. Technol. 603 (2011) 925-930.

[14] M. Schloesser et al., Accuracy of the Laser Raman system for KATRIN, to be published in Proceedings of the International School of Physics "E. Fermi"

[15] W. Gil et al. Status of the Magnets of the Two Tritium Pumping Sections for KATRIN, IEEE Transactions on Applied Superconductivity, vol. 22, issue 3, pp. 4500604-4500604, doi:10.1109/TASC.2011.2175353

[16] O. Kazachenko et al. , TRAP - a cryo-pump for pumping tritium on pre-condensed argon, Nuclear Instruments and Methods in Physics Research A 587 (2008) 136

[17] F. Eichelhardt et al. , First Tritium Results of the KATRIN Test Experiment Trap, Fusion Science and Technology 54 (2008) 615

[18] F.M. Fränkle et al., Radon induced background processes in the KATRIN pre-spectrometer, Astropart. Phys. 353 (2011) 128-134 doi:10.1016/j.astropartphys.2011.06.009.

[19] K. Valerius, The wire electrode system for the KATRIN main spectrometer, Progress in Particle and Nuclear Physics 642 291-293 (2010) doi:10.1016/j.ppnp.2009.12.032. 